\title{
3D Reconstruction with Spiral Computed Tomography in Choroidal Osteoma
}

\author{
Francisco Javier Ascaso ${ }^{1,2}$, Laura Herrera ${ }^{1}$, Laura Villén ${ }^{1}$, Rafael Lasierra ${ }^{3}$, Juan Ibañez ${ }^{1}$, \\ Diana Pérez ${ }^{1}$, José Angel Cristóbal ${ }^{1}$ \\ ${ }^{1}$ Department of Ophthalmology, Lozano Blesa University Clinic Hospital, Zaragoza, Spain; ${ }^{2}$ Aragon Health Sciences Institute, \\ Zaragoza, Spain; ${ }^{3}$ Department of Radiology, Lozano Blesa University Clinic Hospital, Zaragoza, Spain. \\ Email: jascaso@gmail.com; lau141@yahoo.com; villenl@hotmail.com; isa.adiego@gmail.com; juanibanezalperte@msn.com; \\ dianapgpe@hotmail.com; jacristobal@salud.aragon.es
}

Received September $24^{\text {th }}, 2012$; revised October 25 ${ }^{\text {th }}$, 2012; accepted November $2^{\text {nd }}, 2012$

\begin{abstract}
Choroidal osteoma (CO) is a rare, ossifying benign tumor originated in the choroid that typically occurs in otherwise healthy young women $(1,2)$. It is characterized by a yellowish, well demarcated lesion in the juxtapapillary or macular area. The diagnosis is clinical and can be confirmed with the use of fluorescein or indocyanine angiography, optical coherence tomography, computed tomography or magnetic resonance imaging. Choroidal neovascularization or subretinal fluid, the main causes for vision loss, can be treated with laser therapy, photodynamic therapy or intravitreal anti-vascular endothelial growth factor therapy. We present a case of choroidal osteoma, showing the role of the high resolution 3D spiral computed tomography.
\end{abstract}

Keywords: Choroidal Osteoma; 3D Spiral Computed Tomography; Ultrasonography; Fluorescein Angiography

\section{Case History}

A 28-year-old woman complained of gradual decrease in visual acuity in her right eye, which was attributed to a macular lesion. Best-corrected visual acuity in this eye was 20/400. Funduscopy revealed a well-demarcated, slightly elevated, yellow-white to orange lesion located in the juxtapapillary and macular area of the right eye compatible with a choroidal osteoma (CO) (Figure 1).

Fluorescein angiography showed diffuse patchy hyperfluorescence with late-phase generalized tissue staining (Figure 2). B-scan ultrasonography and especially computed tomography (CT) were of particular value to confirm the diagnosis, by showing clearly demonstrable calcium densities in the posterior pole of the affected eye (Figures 3 and 4). A three-dimensional spiral computed tomography was also made to establish the diagnosis (Figure 5).

The patient was unsuccessfully treated with two intravitreal ranimizumab injections.

\section{Discussion}

Choroidal osteoma is a rare, ossifying benign tumor that originates in the choroid. It is usually unilateral and develops in the second or third decade of life, mostly in women [1,2]. Funduscopically, it is characterized by a well-demarcated, slightly elevated, yellow-white lesion

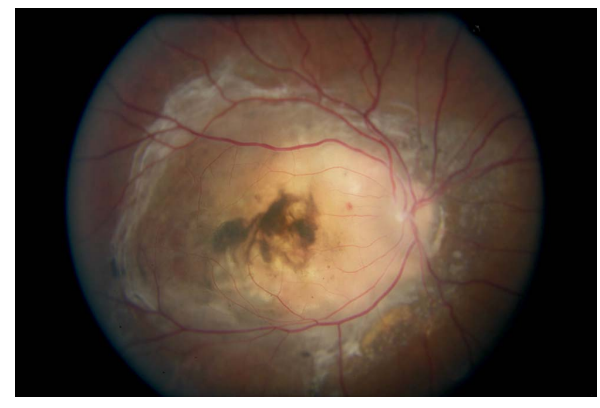

Figure 1. Fundus photograph showing a well-demarcated, slightly elevated, yellow white to orange lesion located in the juxtapapillary and macular area of the right eye compatible with a choroidal osteoma.

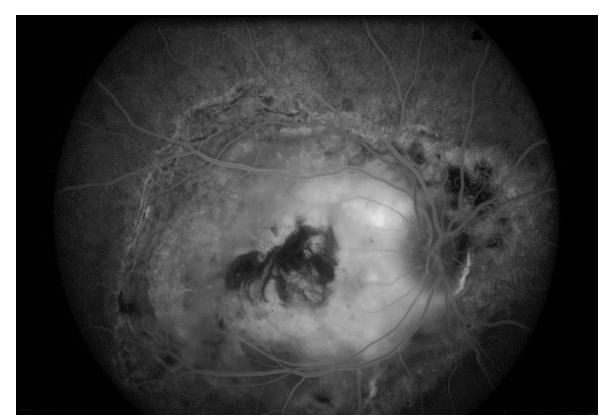

Figure 2. Fundus fluorescein angiography revealed a diffuse patchy hyperfluorescence with late-phase generalized tissue staining. 


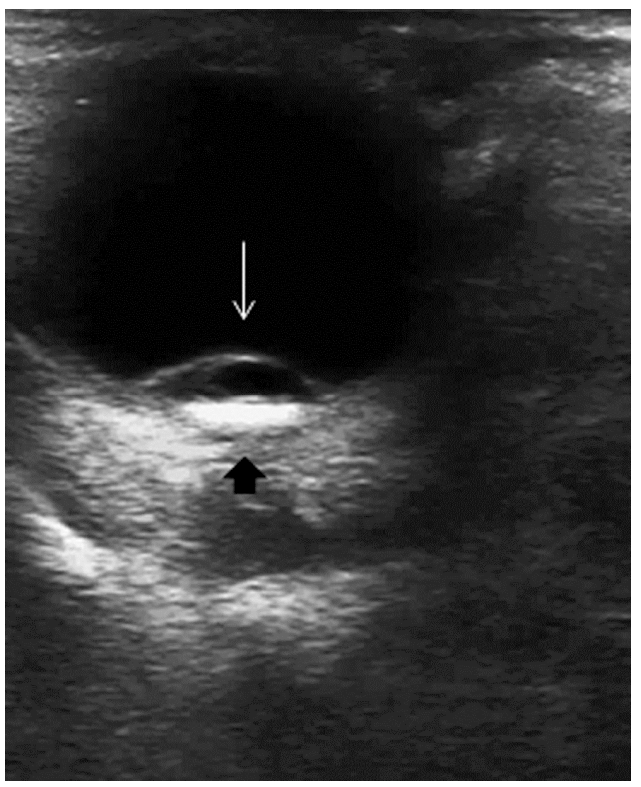

Figure 3. B-scan ultrasonography showing clearly demonstrable calcific densities in the posterior pole of the affected eye (black arrow) and an associated exudative retinal detachment.

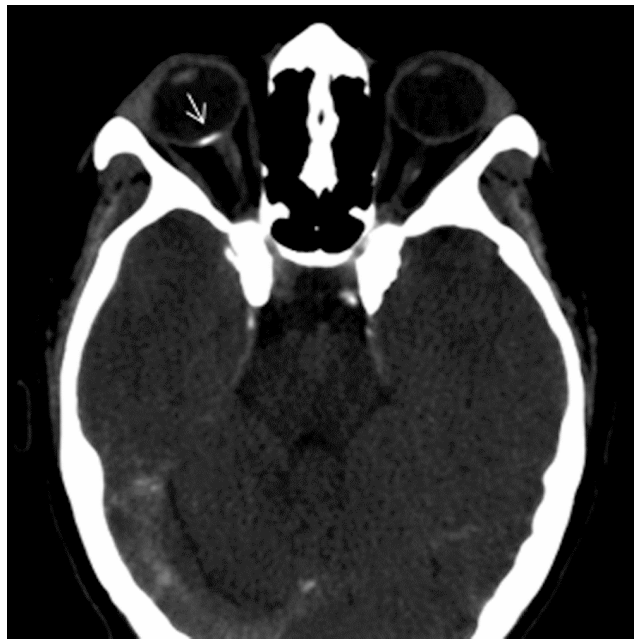

Figure 4. Axial computed tomography (CT) scan reveals a characteristic, well defined, homogeneously radio-opaque plaque of bone density in the posterior wall of the right eyeball, corresponding to a choroidal osteoma (arrow).

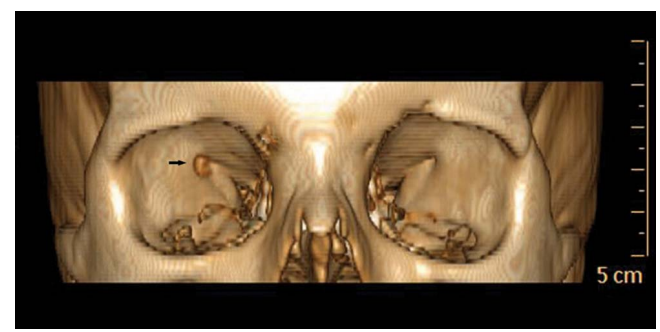

Figure 5. 3D reconstruction of CT scan demonstrating a clearer perception of the extent and location of the tumor (arrow). located in the juxtapapillary or macular area. Fluorescein angiography usually shows diffuse patchy hyperfluorescence with late-phase generalized tissue staining. Optical coherence tomography demonstrates a highly reflective cavernous structure [1,2]. Orbital computed tomographic scan reveals a characteristic, well-defined, homogeneously radio-opaque plaque with the same density as bone at the level of the choroid. This finding enables to distinguish the CO from various dystrophic lesions: atypical or amelanotic malignant choroidal melanoma, metastatic carcinoma, leukemic or lymphomatous infiltrates, choroidal hemangioma, choroidal scars of the macula, and organized subretinal hemorrhage [3,4]. However, multiple diseases are associated with sclerochoroidal calcifications, occurring after inflammatory, infectious, and traumatic injuries to the eye in which there are significant secondary tissue atrophy, degeneration, and necrosis. Likewise, ocular calcification has been reported in hyperparathyroidism, acute and chronic renal failure, sarcoidosis, vitamin D intoxication, and hypercalcemia $[5,6]$.

CT is the first-line imaging modality for orbital evaluation [5]. Nevertheless, conventional radiography has the problem of superimposition, and moreover it is a twodimensional representation of a three-dimensional object. Spiral computed tomography (SCT) may be useful in assessing the size, shape, and location of a CO when compared to the routine CT scan, avoiding potential pitfalls in orbital image interpretation. Compared to CT scan, three-dimensional (3D) SCT is potentially more useful for the diagnosis of CO: a high resolution 3D view of the CO morphology in spatial relation to the surroundding orbital structures is achieved, providing with more details on its size and extension, as well as giving key information to differentiate it form other diseases associated with calcifications. It also helps in the follow-up of patients, by showing highly accurately, whether the CO has responded well to treatment. Disadvantages are its cost, elevated levels of radiation exposure and, to a lesser extent, time requirements and the size of the equipment.

\section{REFERENCES}

[1] F. J. Ascaso and L. Villén, "Fundus Autofluorescence Imaging Findings in Choroidal Osteoma,” Retina, Vol. 31, No. 5, 2011, pp. 1004-1005. doi:10.1097/IAE.0b013e31820d37a6

[2] F. J. Ascaso and R. Lasierra, "Idiopathic Dural Optic Nerve Sheath Calcification Associated with Choroidal Osteoma,” Ophthalmic Surgery, Lasers \& Imaging, 2011 (in Press). doi:10.3928/15428877-20110602-03.

[3] C. L. Shields, B. Perez, M. A. Materin, S. Mehta and J. A. Shieds, "Optical Coherence Tomography of Choroidal Osteoma in 22 Cases: Evidence for Photoreceptor Atrophy over the Decalcified Portion of the Tumor," Ophthalmo- 
logy, Vol. 114, No. 12, 2007, pp. e53-e58. doi:10.1016/j.ophtha.2007.07.037

[4] R. N. Bryan, R. A. Lewis and S. L. Miller, "Choroidal Osteoma,” American Journal of Neuroradiology, Vol. 4, 1983, pp. 491-494.

[5] C. A. LeBedis and O. Sakai, "Nontraumatic Orbital Conditions: Diagnosis with CT and MR Imaging in the Emergent Setting," Radiographics, Vol. 28, No. 6, 2008, pp.
1741-1753. doi:10.1148/rg.286085515

[6] S. Wong, Z. N. Zakov and D. M. Albert, "Scleral and Choroidal Calcifications in a Patient with Pseudohypoparathyroidism,” British Journal of Ophthalmology, Vol. 63, No. 3, 1979, pp. 177-180.

doi:10.1136/bjo.63.3.177 